\title{
Gekommen, um zu bleiben? Der Verbleib internationaler Studierender in Deutschland aus einer Lebenslaufperspektive
}

\author{
Birgit Glorius $^{1}$
}

Eingegangen: 23. Oktober 2015 / Angenommen: 30. Mai 2016 / Online publiziert: 21. Juni 2016

(C) Springer-Verlag Berlin Heidelberg 2016

Zusammenfassung Vor dem Hintergrund des demographischen Wandels in Deutschland werden von der Politik seit geraumer Zeit Bemühungen unternommen, den anhaltenden Fachkräftebedarf durch die Attrahierung hochqualifizierter Zuwanderer zu decken. In diesem Kontext wächst auch das Interesse an internationalen Studierenden als potenziellem Fachkräftenachwuchs für den deutschen Arbeitsmarkt. Deutschland ist ein bedeutendes Zielland für internationale Studierende, und die formalen Rahmenbedingungen für einen Verbleib und Arbeitsmarktzugang nach Studienabschluss wurden in den vergangenen Jahren erheblich liberalisiert. Umfrageergebnisse belegen ein großes Interesse internationaler Studierender an einem Verbleib in Deutschland nach dem Studium, doch die verfügbaren Zahlen zeigen, dass die Bleibeintentionen nur teilweise umgesetzt werden. Dieser Diskrepanz wird in dem vorliegenden Beitrag nachgegangen. Anhand einer qualitativen Fallstudie analysiert der Beitrag die Einstellungen, Erfahrungen und Zukunftsvisionen von internationalen Studierenden in Deutschland unter der Perspektive eines möglichen Verbleibs. Eine besondere Konzentration liegt auf der Frage, wie sich die Verbleibs- oder Wanderungsentscheidung als Teil einer biographischen Statuspassage konstituiert, die am Übergang zwischen Studium und Beruf auftritt. Es zeigt sich, dass lebenslaufbezogene Erwägungen und Standortentscheidungen stark ineinander verwoben sind und auf der Basis eines transnationalen Aktionsraums angestellt werden.

Prof. Dr. Birgit Glorius

birgit.glorius@phil.tu-chemnitz.de

1 Institut für Europäische Studien, Juniorprofessur Humangeographie Ostmitteleuropas, Technische Universität Chemnitz, Thüringer Weg 9, 09126 Chemnitz, Deutschland
Schlüsselwörter Migration von Hochqualifizierten · Lebenszyklus · Studierendenmobilität .

Verbleibsentscheidung

\section{Came to Stay for Good? Mobility Decisions of International Students in Germany from a Lifecourse Perspective}

Abstract Against the background of demographic ageing in Germany, there are increasing attempts to attract high qualified labor force from abroad, and there is high interest to retain international students who graduate from German universities. Germany is an important destination country for international students, and the formal conditions for staying on and entering the labor market upon graduation were largely liberated during the past years. Survey results show a high interest for an extended stay in Germany among international students, but retention rates reveal that those intentions are only partly put into practice. This article investigates on this discrepancy. Using a qualitative case study, the attitudes, experiences and future plans of international students in Germany are analyzed focusing on possible intentions to stay for good. The analysis focuses on the question, how decisions to move or stay on are embedded in biographical status passages such as the transition from university to work life. The results show a strong impact of life cycle considerations with regards to future mobility decisions among the interviewees. Both previous and future migratory decisions are negotiated on the basis of a transnational action space, integrating rational argumentation as well as subjectivities and influences from peers and family members.

Keywords Highly skilled migrants $\cdot$ Life cycle $\cdot$ Student migration $\cdot$ Stay decisions 


\section{Einleitung}

In Deutschland wird ein Mangel an hochqualifizierten Fachkräften beklagt und vor dem Hintergrund der demographischen Alterungsprozesse wird erwartet, dass er sich zukünftig vergrößern wird (Obermeier 2014). Eine Möglichkeit zur Deckung des Fachkräftebedarfs besteht darin, das fehlende Eigenpotenzial durch gezielte Zuwanderung in den deutschen Arbeitsmarkt zu ergänzen. Zahlreiche Politikansätze der vergangenen Jahre waren auf diese Strategie ausgerichtet, doch trotz einer Serie von gesetzlichen Vereinfachungen und speziellen Rekrutierungsprogrammen wird Deutschland von hochqualifizierten Drittstaatenangehörigen nur zögerlich angesteuert (SVR 2011a: 68). Daher stellt sich die Frage, warum das Potenzial der internationalen Studierenden an deutschen Hochschulen nicht entschiedener genutzt wird. Denn diese haben zahlreiche Vorzüge gegenüber Fachkräften, die aus dem Ausland angeworben werden: Sie verfügen über einen anerkannten Bildungsabschluss, sie sind mehrere Jahre in einem deutschsprachigen Lebensumfeld sozialisiert und dürften weniger Integrationsbedarf aufweisen. Der direkte Arbeitsmarkteinstieg in Deutschland könnte eine arbeitsmarktbezogene Sozialisation und damit eine Verstetigung des Aufenthalts fördern. Damit könnten langfristig verbleibende internationale Studierende zur Sicherung des Fachkräftebedarfs und zur Milderung der Folgen der demographischen Alterung in Deutschland beitragen (SVR 2011b: 9).

Dieser Beitrag wendet sich dieser Fragestellung zu und betrachtet die Einstellungen, Erfahrungen und Zukunftsvisionen von internationalen Studierenden in Deutschland unter der Perspektive eines möglichen Verbleibs. Es wird untersucht, welche Faktoren die Entscheidung bezüglich Verbleib, Rück- oder Weiterwanderung beeinflussen und wie der Aushandlungsprozess vor dem Hintergrund bisheriger Migrationserfahrungen und genereller Zukunftsaspirationen abläuft. Ein besonderer Schwerpunkt liegt auf der Frage, wie Überlegungen zum Verbleib bzw. zur Rück- oder Weiterwanderung in biographische Statuspassagen eingebettet werden, die am Übergang zwischen Studium und Beruf auftreten.

Der Beitrag thematisiert im Folgenden die Entwicklung der internationalen Studierendenmigration nach Deutschland auf der Basis der relevanten rechtlichen Rahmenbedingungen (Abschn. 2). Anschließend wird eine theoretische Einbettung des Themas in den Kontext der internationalen Migrationsforschung vorgenommen und der Forschungsstand zu Verbleib und Rückkehr internationaler Studierender dargestellt (Abschn. 3). Auf dieser konzeptionellen Basis werden die oben aufgeworfenen Fragen empirisch beantwortet (Abschn. 4). Dazu wird eine Fallstudie präsentiert, bei der in den Jahren 2008 und 2009 ausländische Studierende und Promovierende an der Martin-Luther-
Universität Halle-Wittenberg mittels qualitativer, fokussierter Interviews zu ihrem bisherigen Bildungs- und Mobilitätsverlauf und ihren Zukunftserwartungen befragt wurden. Abschließend werden die erarbeiteten Befunde nochmals generalisiert und kontextbezogen diskutiert (Abschn. 5).

\section{Entwicklung von Zuwanderung und Verbleib internationaler Studierender}

Die Internationalisierung des Hochschulwesens hat zu einer steigenden Mobilität der Studierenden geführt. Deutschland ist global betrachtet hinter den USA und Großbritannien eines der wichtigsten Gastländer internationaler Studierender (OECD 2014). Im Jahr 2014 waren rund 219.000 bildungsausländische Studierende ${ }^{1}$ an deutschen Hochschulen eingeschrieben, davon rund die Hälfte im Erststudium. Die Hauptherkunftsländer internationaler Studierender in Deutschland waren im Jahr 2014 China (28.381), die Russische Föderation (11.126), Indien (9372), Österreich (9305) und Bulgarien (6741) (DAAD/DZHW 2015).

Während der Studienzugang für EU-Bürger im Rahmen der Freizügigkeit problemlos möglich ist, benötigen internationale Studierende aus Drittstaaten ein Studienvisum und eine Aufenthaltsgenehmigung. Zunächst müssen sie sich für ein Studienprogramm an einer deutschen Hochschule bewerben. Mit der Studienzulassung können sie im Herkunftsland ein Studienvisum beantragen. Hierzu müssen sie nachweisen, dass sie für mindestens ein Jahr selbstständig für ihren Lebensunterhalt aufkommen können. ${ }^{2}$ Nach der Ankunft in Deutschland erhalten sie eine Aufenthaltsgenehmigung zum Zweck des Studiums, die in der Regel auf zwei Jahre befristet ist. Danach muss sie Jahr für Jahr erneuert werden, bis der Zweck des Aufenthaltes erreicht ist. Mit der Aufenthaltsgenehmigung ist eine Arbeitserlaubnis für 90 Arbeitstage im Jahr verbunden, was für viele internationale Studierende ein entscheidender Faktor zur Einkommenssicherung bedeutet (BMI 2014: 61).

Valide Daten zum Verbleib internationaler Studierender in Deutschland nach dem Studienende lassen sich nur schwer generieren, insbesondere hinsichtlich des längerfristigen Aufenthalts. Verbleibsuntersuchungen konzentrieren sich daher entweder auf die Aussagen zu zukünftigen Migrationswünschen seitens der Studierenden oder werten Sekundärdaten aus, wie etwa den Wechsel des Aufenthaltszweckes im Rahmen von Aufenthaltsgenehmigungsverfahren. Da diese im Falle Deutschlands nur für Drittstaaten-

\footnotetext{
${ }^{1}$ Bildungsausländische Studierende sind jene Studierende, die ihre Hochschulzugangsberechtigung an einer Schule im Ausland erworben haben (DAAD/DZHW 2014: 150).

${ }^{2}$ Die Summen richten sich nach den BAFöG-Höchstsätzen und lagen im Jahr 2011 bei 670 Euro monatlich bzw. 8040 Euro pro Jahr (vgl. Mayer/Yamamura/Schneider et al. 2012: 27).
} 
angehörige vorliegen, ergibt sich ein unvollständiges Bild des Bleibeverhaltens internationaler Studierender. So lassen sich die unterschiedlichen Bleibe-Quoten zwischen 23 und 56 Prozent erklären, die in verschiedenen Studien für internationale Studierende in Deutschland berechnet wurden (vgl. DAAD/DZHW 2014: 40; SVR 2015: 17).

Bis vor wenigen Jahren war der Übergang in den Arbeitsmarkt für drittstaatenangehörige Absolventen deutscher Hochschulen äußerst schwierig. Die Aufenthaltserlaubnis erlosch mit dem Studienabschluss. Wenn nicht ein Wechsel des Aufenthaltszweckes (etwa aus familiären Gründen) und der entsprechenden Genehmigung erfolgte, musste der Absolvent in sein Heimatland zurückkehren, um gegebenenfalls von dort einen Arbeitsplatz in Deutschland zu suchen und eine entsprechende Aufenthaltsgenehmigung zu beantragen.

Im Rahmen des neuen Zuwanderungsgesetzes von 2004 wurden Regelungen implementiert, die es ausländischen Hochschulabsolventen aus Drittstaaten sowie aus den damals noch nicht freizügigkeitsberechtigten EU-Staaten ermöglichte, eine einjährige Aufenthaltserlaubnis zum Zweck der Arbeitssuche zu beantragen ( $\$ 16$ Abs. 4 AufenthG). Anfangs wurde diese Regelung kaum genutzt. Im Jahr 2006 besaßen beispielsweise lediglich 1954 von insgesamt 14.860 Hochschulabgängern aus Drittstaaten eine entsprechende Aufenthaltserlaubnis (BMI 2007: 63).

Dieser eher mäßige Erfolg könnte den bürokratischen Barrieren geschuldet gewesen sein, die von Bewerbern und zukünftigen Arbeitgebern überwunden werden mussten: Die Einstellung eines Drittstaatenangehörigen unterlag einer Vorrangprüfung, ob ein Deutscher oder gleichberechtigter Ausländer (EU-Angehöriger oder Schweizer) die ausgeschriebene Position besetzen könnte. Zudem musste ein Jahresmindesteinkommen von 85.500 Euro nachgewiesen werden, um abzusichern, dass es sich bei der Tätigkeit tatsächlich um eine hochqualifizierte Tätigkeit handelte. ${ }^{3}$ Im Jahr 2007 wurde die Vorrangprüfung für Hochqualifizierte abgeschafft, zumindest innerhalb jener Arbeitsmarktsegmente, in denen Fachkräfteengpässe bestanden. Zum 1. Januar 2009 wurde das erforderliche Mindesteinkommen gesenkt und der Arbeitsmarkt für Akademiker aus den neuen EU-Staaten sowie für ausländische Absolventen deutscher Hochschulen vollständig geöffnet; allerdings war weiterhin eine Zustimmung der Bundesagentur für Arbeit erforderlich. Die Zahl der Aufenthaltserlaubnisse zum

\footnotetext{
${ }_{3}^{3}$ Dieses Mindesteinkommen definierte sich durch die Beitragsbemessungsgrenze der gesetzlichen Krankenversicherungen. Der hochqualifizierte ausländische Bewerber musste mindestens das Doppelte dieses Betrages verdienen, im Jahr 2006 85.500 Euro (BMI 2007: 81). Dies ist jedoch für einen Berufsanfänger kaum realistisch. Zum Vergleich: Einer Unternehmensbefragung zufolge lag das durchschnittliche Einstiegsgehalt von Hochschulabsolventen 2006/2007 zwischen 33.000 und 42.000 Euro brutto (Holzapfel 2011).
}

Zweck einer hochqualifizierten Tätigkeit stieg von $4421 \mathrm{im}$ Jahr 2007 auf 7392 im Jahr 2011. Weitere Erleichterung brachte die Umsetzung der EU-Hochqualifiziertenrichtlinie (2009/50/EG) zum 1. August 2012. Unter anderem wurden die Laufzeit der Aufenthaltsgenehmigung zum Zweck der Arbeitsuche von 12 auf 18 Monate verlängert, die Einkommensgrenze weiter abgesenkt und der Zugang zur dauerhaften Niederlassungserlaubnis erleichtert (BMI 2008: 89; BMI 2014: 64 ff.).

Trotz dieser verbesserten Rahmenbedingungen scheint das Verbleibspotenzial internationaler Hochschulabsolventen in Deutschland noch nicht ausgeschöpft zu werden. So schlossen beispielsweise im Studienjahr 2014 insgesamt 43.671 bildungsausländische Studierende ein Hochschulstudium in Deutschland ab, davon 30.429 aus Drittstaaten. Etwa 23.800 dieser Absolventen kamen für eine Aufenthaltsgenehmigung zur Arbeitsplatzsuche ( $\$ 16$ Abs. 4 AufenthG) in Frage. Zum Jahresende 2014 waren jedoch lediglich 7120 Personen mit einer entsprechenden Aufenthaltsgenehmigung registriert (BMI 2016: 82 f.).

\section{Bleibewahrscheinlichkeit und Einflüsse auf das Bleibeverhalten}

Die Frage, was die längerfristige Niederlassung einer Person an einem gegebenen Standort beeinflusst, wird von der raumbezogenen Sozialforschung kaum beleuchtet. Auch innerhalb der Migrationsforschung wird diese Frage selten in den Mittelpunkt gerückt, was sicherlich dem lange Zeit vorherrschenden Paradigma der Einmaligkeit und Endgültigkeit von Migrationsentscheidungen geschuldet ist (Boyd 1989: 640; Pries 2001: 32). Doch selbst im Kontext aktueller Forschungsparadigmen, die Migranten in einen transnationalen Sozialraum eingebettet konzeptualisieren, werden Bleibeentscheidungen im Gegensatz zu Mobilitätsentscheidungen wenig thematisiert.

Im Zusammenhang mit der internationalen Mobilität von Hochqualifizierten und Studierenden steht häufig die Frage des Humankapitaltransfers im Fokus. Die entsprechenden Studien verwenden Ansätze ökonomischer HumankapitalTheorien in Verbindung mit dem von Bourdieu (1986) entwickelten Begriff des kulturellen Kapitals. Anfangs standen der vermutete Entzug von Humankapital (brain drain) durch Emigration und die negativen Folgen für weniger entwickelte Staaten im Vordergrund (vgl. z. B. Baldwin 1970; Galinski 1986). Später wurde auch die Möglichkeit des Rückerwerbs von Humankapital durch Remigration der ehemaligen Studierenden in Betracht gezogen und der Einfluss von Rückwanderern auf die regionale Entwicklung in ihrem Herkunftsland untersucht (z. B. Müller 2007; Le Bail/Shen 2008; Saxenian 2008). Mehrere Studien thematisierten den Wert von Auslandserfahrung für die berufliche 
Integration rückkehrender Graduierter im europäischen Migrationsraum (z. B. Baláž/Williams 2004; Teichler/Janson 2007; Wolfeil 2012).

Einige Studien betonen die Bedeutung des räumlich gebundenen sozialen Kapitals für Mobilitätsentscheidungen internationaler Studierender und Akademiker, insbesondere für einen erfolgreichen Arbeitsmarkteinstieg nach dem Studienabschluss. So kann der migrationsbedingte Verlust sozialen Kapitals im Herkunftsland Rückwanderungsentscheidungen negativ beeinflussen (Pichler/Prontera 2012: 97). In einer Studie zur Mobilität internationaler Wissenschaftler in Deutschland weist Neusel (2012: 24) auf den starken Einfluss beruflicher Netzwerke für die Gestaltung wissenschaftlicher Karrieren hin und nimmt diesbezüglich Nachteile für die zugewanderten Wissenschaftler und insbesondere die Wissenschaftlerinnen an. Auch (antizipierte) Diskriminierung auf dem Arbeitsmarkt oder die negative Einschätzung der individuellen oder allgemeinen ökonomischen Perspektiven können eine Rolle für Migrationsentscheidungen spielen, wie in Studien zur Abwanderungsbereitschaft türkeistämmiger Hochschulabsolventen in Deutschland herausgearbeitet wurde (Sezer/Dağlar 2009: 23 ff.).

Die Wahrscheinlichkeit eines Verbleibs internationaler Studierender im jeweiligen Gastland wurde in verschiedenen Studien in Gegenüberstellung zur Rückkehroption untersucht und anhand der jeweils vorliegenden Datensätze statistisch modelliert (Chiu/Hou 2007; Soon 2008; Gibson/ McKenzie 2009). Dabei ergeben sich folgende Erkenntnisse: Die Rückkehrwahrscheinlichkeit wird positiv beeinflusst durch die Existenz von Sozialkapital im Herkunftsland, während Sozialkapital im Gastland einen weiteren Verbleib unterstützt (Gibson/McKenzie 2009: 32 ff.). Die Aufenthaltsdauer im Gastland ebenso wie die Wertigkeit des erworbenen Bildungsabschlusses wirken sich positiv auf den Verbleib aus. Chiu/Hou (2007: 10) erklären dies mit guten Arbeitsmarktplatzierungen und dem hohen Risiko, im Falle einer Rückkehr Rückschritte in der Karriereentwicklung zu erleiden. In der Studie von Soon (2008) zu Rückkehrintentionen internationaler Studierender in Neuseeland zeigte sich eine ursprünglich geäußerte Rückkehrintention als wichtigste erklärende Variable für die spätere Rückkehr. Weitere Einflussfaktoren für eine Rückkehr- bzw. Bleibeentscheidung resultierten aus vergleichenden Einschätzungen der Studierenden zu Aspekten wie Gehaltsniveau, Arbeitsatmosphäre, Möglichkeiten der Wissensanwendung sowie Lebensstil in Herkunfts- und Gastland (Soon 2008: 10). Diese Gegenüberstellung zeigt bereits, dass die Frage des Verbleibs internationaler Studierender idealerweise auf der Mikroebene und aus einer transnationalen Perspektive betrachtet werden sollte. Dabei sind weniger die objektiven Bedingungen in den zur Entscheidung stehenden Ländern von Interesse, sondern vielmehr die subjektive Evaluierung dieser Bedingungen durch die internationalen Studierenden.

Mehrere Studien aus der angewandten Forschung untersuchten in den vergangenen Jahren die Bleibewahrscheinlichkeit und mögliche Einflussfaktoren auf Verbleibsentscheidungen bei internationalen Studierenden in Deutschland (Heß 2009; SVR 2011b; Mayer/Yamamura/Schneider et al. 2012; SVR 2012; Hanganu/Heß 2014; SVR 2015). Dabei wurde - bei aller Vorsicht hinsichtlich der Validität von prognostischen Selbstaussagen - ein großes Verbleibspotenzial deutlich: So gaben in einer 2011 durchgeführten Online-Befragung unter Studierenden aus Drittstaaten 80 Prozent der Master-Studierenden und 67 Prozent der Doktoranden an, nach dem Studienabschluss in Deutschland bleiben $\mathrm{zu}$ wollen - allerdings eher mittel- als langfristig (SVR 2012: 38). ${ }^{4}$ Fast die Hälfte der Befragten sah gute Chancen, einen adäquaten Arbeitsplatz in Deutschland zu finden. Eine mögliche berufliche Weiterentwicklung wurde als wichtigstes Kriterium für einen Verbleib genannt, wobei auch geäußert wurde, dass diese Frage nur im Kontext der jeweiligen sozialen und familiären Einbindung beantwortet werden kann. ${ }^{5}$ Im Vergleich zur Gesamtheit der Befragten waren die Bleibewilligen eher jünger, hielten sich bereits längere Zeit in Deutschland auf, waren eher in Master-Studiengängen zu finden als in Promotionsprogrammen und studierten häufiger MINT-Fächer ${ }^{6}$ als Sozial- oder Kulturwissenschaften. Die Autoren der Studie resümieren, dass eine Arbeitsmarktintegration in Berufen, in denen kulturspezifisches Wissen oder gute Deutschkenntnisse eine große Rolle spielen, offensichtlich schwerer vorstellbar sei als in den technischen Berufen (SVR 2012: 40). Diese Interpretation wird unterstrichen durch die Selbsteinschätzung der Befragten, die den Mangel an deutschen Sprachkenntnissen als großes Problem hinsichtlich eines mittelfristigen Verbleibs und eines gelingenden Arbeitsmarktzuganges ansehen. Zum Zeitpunkt der Befragung wurden die eigenen Deutschkenntnisse von 37 Prozent als nur elementar (Stufe $\mathrm{A} 1 / \mathrm{A} 2)^{7}$ eingeschätzt. Dieser Befund lässt sich durch die Verbreitung englischsprachiger Studienangebote erklären, die den Erwerb deutscher Sprachkenntnisse auf einem guten Niveau nicht erforderlich machen (SVR 2012: 48 f.).

\footnotetext{
${ }^{4}$ Nur 12 Prozent der Befragten gaben an, länger als fünf Jahre bleiben zu wollen (SVR 2012: 38).

5 Immerhin war fast ein Viertel zum Zeitpunkt der Befragung verheiratet und muss dementsprechend eine Migrations- oder Bleibeentscheidung nicht nur für sich, sondern für und mit dem Partner treffen (SVR 2012: 33)

${ }^{6}$ Mathematik, Informatik, Naturwissenschaft, Technik.

7 Der gemeinsame Europäische Referenzrahmen für Sprachen gliedert sprachliche Kompetenzen in sechs Stufen von A1 (Anfänger) bis C2 (Experten). Als Minimalanforderung für Studium und Beruf wird das Niveau B1 betrachtet, für qualifizierte Berufe oder jene mit einem hohen Kommunikationsanteil (z. B. Ärzte, Pflegekräfte) wird das Niveau B2 als erforderlich betrachtet (Sheils 2001).
} 
Ein weiterer Forschungsstrang befasst sich aus einer lebenslaufbezogenen Perspektive mit den Mobilitäts- und Bleibeentscheidungen internationaler Graduierter (z. B. Alberts/Hazen 2005; Szelényi 2006; Geddie 2013). Dabei wird insbesondere auf das transitorische Stadium im Kontext des Studienabschlusses Bezug genommen. Eine Mobilitäts- oder Bleibeentscheidung ist demnach eng verknüpft mit der weiteren Karriereplanung, aber ebenso mit Partnerschafts- bzw. familienbezogenen Aspekten. Im Falle internationaler Graduierter wird dieser Entscheidungsprozess noch komplexer durch das transnationale Setting, in dem sie sozial verortet sind. So zeigt beispielsweise Geddie (2013) in ihrer Untersuchung internationaler Studierender in London (Großbritannien) und Toronto (Kanada) die Komplexität der Lebenssituationen und die starken Einflüsse transnationaler sozialer Bindungen auf die weiteren Migrations- und Karrierestrategien. Mobilitätsentscheidungen wurden nicht primär aus ökonomischer Ratio oder Karrieremotiven getroffen und sie betrafen nicht lediglich die Frage Verbleib versus Rückkehr. Vielmehr wurden Fragen der sozialen Verantwortung beispielsweise gegenüber Geschwistern und Eltern sowie Fragen einer Doppelkarriere (dual career) im Rahmen der (vielfach ebenfalls international angelegten) Partnerschaft in die Entscheidung mit einbezogen.

Aus der Gesamtheit der wissenschaftlichen Erkenntnisse der Hochqualifiziertenforschung und zur Mobilität internationaler Studierender lassen sich als Zwischenfazit drei Ableitungen treffen: Die Frage des Verbleibs oder der Weiterwanderung einer mobilen Population lässt sich keinesfalls mit Ansätzen beantworten, die Migrationsentscheidungen als einmalige und endgültige Entscheidungen annehmen. Vielmehr ist davon auszugehen, dass vergangene und aktuelle Mobilitätserfahrungen die Entscheidungsbasis für weitere Mobilitätsentscheidungen nachhaltig verändern und dass den Entscheidungen ein transnationaler Bezugsrahmen zugrunde gelegt wird. Eine duale Untersuchungsstruktur, die lediglich (dauerhaften) Verbleib versus (dauerhafter) Rückkehr untersucht, zielt deshalb zu kurz.

Zweitens lässt sich ableiten, dass Mobilitäts- oder Bleibeentscheidungen nicht ausschließlich aus ökonomischer Ratio heraus getroffen werden. Vielmehr ist die (transnationale) soziale Einbindung der Akteure zu berücksichtigen, wie auch die Unvollkommenheit der Informationsbasis und die Selektivität und Subjektivität hinsichtlich der Wahrnehmung und Verarbeitung entscheidungsrelevanter Informationen.

Schließlich ist hinsichtlich der hier im Mittelpunkt stehenden Personengruppe auch ihre Stellung im Lebenslauf entscheidend: Der Studienabschluss stellt eine wichtige Statuspassage dar; während dieser Zeit werden viele Entscheidungen gleichzeitig getroffen (z. B. Berufseinstieg, Umzug bzw. Migration sowie Familiengründung), die sich dementsprechend auch gegenseitig beeinflussen.

\section{Verbleib in Deutschland aus der Perspektive internationaler Studierender und Wissenschaftler}

Die bisherigen Erörterungen haben gezeigt, dass das deutsche Zuwanderungsgesetz zusammen mit den konjunkturellen Bedingungen gute Voraussetzungen für einen längerfristigen Verbleib internationaler Studierender in Deutschland bietet. Warum von dieser Möglichkeit dennoch vielfach nicht Gebrauch gemacht wird, wird nun im Rahmen einer Fallstudie weiter erörtert, die in den Jahren 2008 und 2009 an der Martin-Luther-Universität Halle-Wittenberg durchgeführt wurde. Die Grundgesamtheit der Studie umfasste 1392 internationale Studierende im Erststudium sowie 240 in Promotionsprogrammen (Martin-Luther-Universität 2008). Es fanden insgesamt 31 themenzentrierte qualitative Interviews mit internationalen Studierenden, Promovierenden und Postdoktoranden statt. Die Interviewpartner wurden durch ein Schneeballverfahren gewonnen, das seinen Anfang in Deutschkursen und Freizeitprogrammen für internationale Studierende hatte. Auch die Homepage des Akademischen Auslandsamtes warb für die Untersuchung. Im Ergebnis konnte eine breite Streuung hinsichtlich Geschlecht, Herkunftsregion, Studienfach und Abschlussart erzielt werden (vgl. Tab. 1).

Es wurde ein biographischer Zugang gewählt mit dem Ziel zu verstehen, wie zukünftige Migrationsentscheidungen vor dem Hintergrund bisheriger Migrationserfahrungen ausgehandelt werden. Gemäß dieses Zugangs wurde ein Leitfaden entwickelt, der als Gesprächsimpuls zur Reflexion der zurückliegenden Migrationsentscheidung aufforderte. Die thematischen Hauptblöcke des Leitfadens umfassten Fragen zur Migrationsentscheidung und der Realisierung der Migration sowie des Einlebens und der Integration vor Ort. Der daran anschließende thematische Block zu den $\mathrm{Zu}$ kunftsperspektiven forderte eine Begründung auf der Basis der bisherigen Mobilitäts- und Integrationserfahrungen. Die Interviews wurden aufgezeichnet, transkribiert und in einem mehrstufigen Verfahren analysiert, das in den Bereich der narrativen Einzelfallanalyse sowie der qualitativen Inhaltsanalyse eingeordnet werden kann (Flick 2007: $473 \mathrm{ff}$.). Das Ziel dieser - anscheinend gegensätzlichen - Analysewege war es, einzelne für das Thema wichtige Aspekte sowohl in ihren unterschiedlichen Ausprägungen betrachten zu können (thematische Querschnittsanalyse) als auch die Ausprägungen im Kontext der jeweiligen Lebensgeschichte begründen zu können (biographische Längsschnittanalyse).

Der nun folgende Ausschnitt aus der Gesamtheit des analysierten Materials konzentriert sich auf drei Schwerpunkte: das Nachvollziehen der ursprünglichen Migrationsentscheidung als Grundlage für zukünftige Standortentscheidungen (Abschn. 4.1), die Reflexion der Umgebungsbedingungen im Hinblick auf einen längerfristigen Verbleib 
Tab. 1 Charakterisierung der Interviewpartner

\begin{tabular}{|c|c|}
\hline Aspekt & Verteilung nach Untergruppen \\
\hline Studienprogramm & $\begin{array}{l}\text { Bachelor: } 9 \\
\text { Diplom/Master: } 10 \\
\text { Promotion: } 10 \\
\text { Post-Doc: } 2\end{array}$ \\
\hline Studiengebiet & $\begin{array}{l}\text { Sprach- und Kulturwissenschaften: } 7 \\
\text { Sport: } 1 \\
\text { Rechtswissenschaften/Wirtschafts- und Sozi- } \\
\text { alwissenschaften: } 9 \\
\text { Mathematik/Naturwissenschaften: } 11 \\
\text { Ingenieurswissenschaften: } 3\end{array}$ \\
\hline Alter & $\begin{array}{l}22 \text { Jahre und jünger: } 9 \\
\text { 23-26 Jahre: } 9 \\
\text { 27 Jahre und älter: } 13\end{array}$ \\
\hline Geschlecht & $\begin{array}{l}\text { männlich: } 15 \\
\text { weiblich: } 16\end{array}$ \\
\hline Familienstand & $\begin{array}{l}\text { ledig: } 25 \\
\text { verheiratet: } 6 \text { (davon } 2 \text { mit Kind/Kindern) }\end{array}$ \\
\hline Herkunftsregion & $\begin{array}{l}\text { Asien: } 8 \\
\text { Afrika/Naher Osten: } 4 \\
\text { Westeuropa: } 2 \\
\text { Mittel- und Südosteuropa: } 8 \\
\text { Russland: } 4 \\
\text { USA: } 1 \\
\text { Süd- und Mittelamerika: } 4\end{array}$ \\
\hline
\end{tabular}

(Abschn. 4.2) sowie die Einbettung von vergangenen und zukünftigen Standortentscheidungen in die Gesamtbiographie (Abschn. 4.3).

\subsection{Die Entscheidung zur Migration nach Deutschland}

Ein erster Schritt im Erkenntnisprozess, wie Verbleibsentscheidungen nach dem Studienabschluss zustande kommen könnten, ist das Nachvollziehen der ursprünglichen Migrationsentscheidung aus dem Kontext der individuellen (Migrations-)Biographie. Die Erörterungen der Interviewpartner zu ihrer Entscheidung für den aktuellen Studienstandort zeigen eine mehrstufige Entscheidungsfindung. Am Anfang stand der Entscheidungsprozess für einen Auslandsaufenthalt als Solches, noch ohne den Fokus auf ein spezifisches Zielland zu setzen. Verhandelt wurde hierbei die Frage, welche Vorteile der Auslandsaufenthalt gegenüber einem Verbleib im eigenen Land haben könnte. Die beiden Hauptargumente für einen Auslandsaufenthalt waren die schlechte Ausbildungsqualität bzw. der begrenzte Zugang zu bestimmten Studiengebieten oder Universitätsstandorten im Heimatland sowie das Argument, dass ein Auslandsaufenthalt förderlich für die weitere berufliche und Persönlichkeitsentwicklung sein könnte. Letzteres Argument fokussierte überwiegend auf den Erwerb von interkulturellen Kompetenzen und wurde vor allem von Interviewpartnern aus asiatischen Herkunftsländern vorgebracht.

Erst nachdem der Entschluss zu einem Auslandsaufenthalt getroffen war, wurden verschiedene Zielregionen in
Erwägung gezogen. Die Entscheidung für Deutschland als Zielland und Halle an der Saale als Studienstandort fiel meist in einer Kombination aus rationalen Erwägungen und der Einflussnahme sozialer Netzwerke. Wichtige Aspekte im Rahmen der Standortevaluierung waren die jeweiligen Studien- bzw. Forschungsschwerpunkte, das Vorhandensein englischsprachiger Studienprogramme, der Ruf der Hochschule im gewählten Bereich sowie weiche Standortfaktoren, wobei für Halle vor allem die niedrigen Lebenshaltungskosten sprachen.

Die meisten Interviewpartner besaßen bereits Migrationserfahrungen. Diese reichten von Studien- und Forschungsaufenthalten in verschiedenen Ländern über Schüleraustausch oder Jugendaustausch in Deutschland bis hin zu familiären Bezügen ins Ausland bzw. direkt in den deutschsprachigen Raum. Insgesamt fünf der Interviewpartner hatten in ihrem Heimatland eine deutsche Schule besucht, was den Weg in den deutschsprachigen Raum bereits vorzeichnete.

Während die Graduierten meist durch das Angebot an Promotionsmöglichkeiten und Forschungsschwerpunkten an die Martin-Luther-Universität in Halle kamen, waren es bei den Studierenden im Erststudium überwiegend Hinweise aus sozialen Netzwerken, die mit entsprechenden persönlichen Informationen oder Referenzen die Entscheidung strukturierten. Häufig gab es auch bereits persönliche Anknüpfungspunkte, wie frühere Gastfamilien aus dem Schüleraustausch, zu denen noch Kontakt bestand, oder Landsleute, die sich ebenfalls für Deutschland bzw. Halle entschieden hatten. Auch Eltern und Verwandte hatten einen Anteil daran, dass die Entscheidung zugunsten Deutschlands ausfiel. Die Wirkweise dieser Einflüsse lässt sich anhand der folgenden Zitate gut nachvollziehen:

StudM3 $^{8}$, Student der Betriebswirtschaftslehre aus China: „Okay, ich war früher bei einem Austauschprogramm in der Schule und bin dadurch an eine Schule nach Deutschland gekommen und hab' auch bei einer deutschen Familie gelebt. Deswegen kannte ich schon Deutschland als ein gutes Land zum Studieren, weil es auch eine sehr hohe Ausbildungsqualität gibt und eine schöne Atmosphäre zum Studieren."

StudM9, Sportstudent aus Ecuador, hatte bereits durch seine Eltern Bezüge zum deutschsprachigen Raum. Die Familie lebte einige Zeit in Österreich, da sein Vater dort seine Facharztausbildung absolvierte. Seine Entscheidung, in Deutschland zu studieren, sieht der Student in der Rückschau stark durch die positive Erfahrung und Unterstützung seiner Eltern beeinflusst: „Also meine Eltern auf jeden Fall, weil die hatten die Erfahrung gemacht in Österreich und die

\footnotetext{
${ }^{8}$ Die Pseudonyme unterscheiden nach dem Status als Studierende (Stud) und Graduierte (Grad) sowie nach Geschlecht (M: männlich, W: weiblich) und enthalten eine fortlaufende Ordnungsnummer.
} 
meinten so, O.K. schön, schöne Platz zu studieren und alles, und ich glaube ich verwirkliche den Traum meines Vaters hier, also ich glaube, er wollte, dass ich hier bleibe. Sagen wir nicht für immer, aber für die nächsten 20 Jahre oder so. Also dass ich meine Karriere hier mache."

Ähnlich äußerten sich viele der Befragten. Ein wesentlicher Befund ist zudem, dass keiner der Interviewpartner eine definitive Verbleibsabsicht äußerte, und auch hinsichtlich einer Rückkehr gab es wenige eindeutige Aussagen. Dies widerspricht Ergebnissen aus Studien, in denen hohe Anteile der Befragten definitive Rückkehr- bzw. Verbleibsabsichten äußerten (z. B. Soon 2008; SVR 2012) und wirft Fragen hinsichtlich der Validität der verschiedenen Befragungsmethoden auf, die an dieser Stelle jedoch nicht beantwortet werden können.

\subsection{Reflexionen über den aktuellen Auslandsaufenthalt als Voraussetzung für weitere Standortentscheidungen}

In einem zweiten Schritt wurden die Reflexionen der Interviewpartner zu ihrem aktuellen Auslandsaufenthalt nachvollzogen. Diesem Erkenntnisschritt lag die Annahme zugrunde, dass vorangegangene Migrationserfahrungen und ihre mentale Verarbeitung entscheidende Komponenten im Aushandlungsprozess zukünftiger Standortentscheidungen darstellen. Konkret wurde die Hypothese formuliert, dass die Zufriedenheit mit dem aktuell erlebten Aufenthalt in Deutschland und das Gefühl der sozialen Integration wichtige Voraussetzungen für die Entscheidung hinsichtlich des weiteren Verbleibs sind. Betrachtet man die gesammelten Daten unter diesem Aspekt, wird unmittelbar der Unterschied zwischen Studierenden im Erststudium und den Teilnehmern an (englischsprachigen) Master- und Promotionsprogrammen deutlich.

Die Teilnehmerinnen und Teilnehmer an Promotionsprogrammen, welche häufig in Forschungsinstituten auf dem Campus angesiedelt sind, halten sich überwiegend innerhalb dieses (internationalen) Wissenschaftlerumfeldes auf. Vielfach erhalten sie innerhalb der Institute die notwendige Unterstützung, um die ersten Schritte in Deutschland zu gehen, wie z. B. Wohnungssuche, Eröffnung eines Bankkontos oder die Meldung im Einwohnermeldeamt. Die Einbindung in die Forschercommunity und die institutionelle Unterstützung werden sehr gelobt, dennoch vermissen viele Interviewpartner Sozialkontakte außerhalb des Arbeitsplatzes, um Gefühle der Einsamkeit oder des Heimwehs zu bekämpfen. „Yes, homesickness is there, because you have, it's natural, everybody misses his family, his friends, and other things, and here we are spending much of our time in the laboratory" (GradM3, Pharmazeut, Doktorand aus Äthiopien).
Studierende im Erststudium hingegen sind in der Regel dazu gezwungen, sich von Anfang an selbstständig in einer deutschsprachigen Lebens- und Studienumgebung zu behaupten. Nach der Überwindung von anfänglichen Schwierigkeiten führt dies zu einer schnellen Integration in ein deutschsprachiges Umfeld und zur Erschließung lokaler sozialer Netzwerke. So war beispielsweise StudW1 aus Rumänien die einzige Ausländerin in ihrem Studiengang Bioinformatik. Anfangs kostete es sie Überwindung, auf die deutschen Kommilitonen zuzugehen, doch inzwischen hat sie einen großen Freundeskreis in Halle und fühlt sich sehr wohl: „In meinem [Studien-]Jahr bin ich die Einzige, die Ausländerin ist. So, deshalb hatt' ich von Anfang an mit Deutschen zu tun, das heißt, sind all meine Freunde hier prinzipiell deutsch." Auch Studentenjobs sind eine Möglichkeit, den Schritt aus dem universitären Umfeld hinaus ins Alltagsleben zu gehen, den lokalen Bekanntenkreis zu erweitern und dabei ein Gefühl der Zugehörigkeit zu entwickeln, das emotional stabilisierend wirkt. So berichtet StudM9, Sportstudent aus Ecuador, von seinem Studentenjob als Barkeeper: „Durch die Arbeit, wie gesagt, hab’ ich die Stammgäste kennengelernt. Dann hab' ich ein bisschen von der spanisch-deutschen Kneipenszene kennengelernt. [...]. Ich komme zum Beispiel nach der Arbeit ins ,Flower Power" ... Hallo, hallo, hallo ... und begrüßen mich auch so und ich fühle mich einfach gut ... Ja, die kennen mich dann halt und das ist einfach nur ein gutes Gefühl.“

Aufgrund dieser lokalen Verankerung scheinen Studierende im Erststudium besser sozial integriert $\mathrm{zu}$ sein als die Nachwuchswissenschaftler. Ein weiterer bedeutender Unterschied zwischen beiden Gruppen sind die Sprachkenntnisse. Während die Studierenden in der Regel ein bis zwei Semester das (deutschsprachige) Studienkolleg besuchen, um die Studienzulassung zu erhalten, bewegen sich viele Doktoranden überwiegend in einem englischsprachigen Arbeitsumfeld und finden kaum Gelegenheiten, einen Deutschkurs zu belegen. Vielfach sind sie in Alltagssituationen auf einen Übersetzer angewiesen. Diese Sprachbarriere im Alltag wird von ihnen selbst als wesentliches Integrationshindernis betrachtet: „My big problem is the language. I mean, I cannot go to the shops to buy, for example, furniture. I need help from other persons that can speak German. I haven't enough time to go to the [language] class, but this is my major, my most important problem, I think“ (GradW6, Ingenieurin, Doktorandin aus dem Iran).

Hinsichtlich des Einflusses ihrer aktuellen Migrationserfahrungen auf weitere Mobilitätsentscheidungen unterschieden die Interviewpartner klar zwischen kurz- und mittelfristigen gegenüber längerfristigen oder gar dauerhaften Aufenthalten. Bezogen auf kürzere Aufenthalte (2-3 Jahre) loben sie den aktuellen Standort, wobei sie die gut ausgebaute Forschungslandschaft, das angenehme Lebensumfeld und die vielfältigen kulturellen Angebote hervorheben. 
Bezogen auf einen längerfristigen Aufenthalt geraten allerdings andere Aspekte in den Fokus: Abgesehen von den Schwierigkeiten, den Alltag ohne gute Deutschkenntnisse zu meistern, wird auch die deutsche Mentalität, die als kühl und zurückhaltend wahrgenommen wird, als problematisch empfunden. Vielfach wurde auch die Empfindung geäußert, dass Deutschland kein klassisches Einwanderungsland sei, was sich in mangelnder ethnischer Diversität und Dominanz der Monolingualität zeige. Um diese Einschätzung zu begründen, ziehen viele Interviewpartner Erfahrungen aus anderen Auslandsaufenthalten heran, wie das im Zitat von GradM2 (Geograph, Post-Doktorand) aus China zum Ausdruck kommt: „You know, I have this story, once I wanted to open a bank account, so it should be an easy job in the U. S. or somewhere else, even when I barely spoke a little English when I first got to the U. S., but still I managed to go through that. But here it's very difficult. I went there three times. Last time I went with my colleague, and she is German. And that, you know, is a very unfriendly environment for a foreigner."

Unzufrieden sind auch viele mit der Transparenz von Arbeits- und Aufenthaltsmöglichkeiten, nicht nur was einen zukünftigen Verbleib anbelangt, sondern auch bezogen auf den aktuellen Aufenthalt. Oft verfügen sie nur über lückenhafte und veraltete Informationen, und aus den Interviews wird deutlich, dass vielfach die im „Behördendeutsch“ gegebenen Auskünfte nicht hinreichend verstanden werden. Auf diese Weise entstehen Fehlinformationen und falsche Einschätzungen, die ebenfalls zukünftige Entscheidungen beeinflussen.

Hinsichtlich einer potenziellen Binnenmobilität innerhalb Deutschlands nach dem Studienabschluss sind die Beschäftigungsmöglichkeiten ausschlaggebend, die für spezifische Professionen in Halle unterschiedliche Perspektiven eröffnen. Während Absolventen der Sprach- oder Kulturwissenschaften sich bereits sehr frühzeitig auf größere Ballungsräume fokussierten, ergaben sich für die naturwissenschaftlich orientierten Studierenden wissenschaftliche Anschlussmöglichkeiten an der Martin-Luther-Universität, die auch gerne in Anspruch genommen wurden. Kenntnisse zur Arbeitsmarktlage in der Region oder konkrete Unternehmenskontakte, die in eine spätere Beschäftigung münden könnten, waren demgegenüber nicht vorhanden.

Insgesamt wussten die Interviewpartner wenig über die regionalen Verschiedenheiten in Deutschland. Sie konnten zwar die wesentlichen Unterschiede im Bereich Einkommen, Wohnungsmarkt sowie die deutsch-deutsche Vergangenheit wiedergeben und waren vor ihrer Ankunft teilweise vor dem ostdeutschen Standort Halle gewarnt worden, da die Ausländerfeindlichkeit dort höher sei. Diese Warnungen deckten sich jedoch nicht mit ihren eigenen Erfahrungen als Ausländer in Halle, die überwiegend neutral bis positiv waren. Mögliche Diskriminierungen aufgrund der Herkunft würden dementsprechend für ganz Deutschland angenommen, wie aus der Narration einer ägyptischen Studentin hervorgeht, die aus religiösen Gründen ein Kopftuch trägt: „Und nach der Uni, ich will eigentlich in Deutschland bleiben. Obwohl ich weiß, ohne Master habe ich keine Chancen in Deutschland zu arbeiten. Und mit Kopftuch schon gar nicht. [...] Aber ich hab' immer noch Hoffnung. Genauso wie ich diesen Job bei der Sparkasse gefunden hab', obwohl es auch natürlich nicht erlaubt ist mit Kopftuch. Aber naja, die haben gemerkt ich hab' Fähigkeiten, ich habe viele Vorteile, ich bin qualifiziert genug, dass ich da arbeiten kann. Und, na ja, ich kann ja auch viele Sprachen, ich mag auch die Sprachen und so deswegen" (StudW6, Bachelor Wirtschaft/Politik).

Zusammenfassend lässt sich sagen, dass die Interviewpartner sehr zufrieden mit ihrer Standortentscheidung sind, allerdings nur bezogen auf einen vorübergehenden Aufenthalt. Dabei stehen die Studien- und Forschungsbedingungen im Vordergrund. Wenn ein langfristiger Verbleib in Erwägung gezogen wird, nehmen Fragen der sozialen Integration an Bedeutung zu. Mangelnde eigene Deutschkenntnisse, aber auch eine empfundene fehlende Diversität und Weltoffenheit im deutschen Alltag gewinnen dann stärker an Gewicht. Fehlende Transparenz hinsichtlich der formalen Möglichkeiten eines Verbleibs verstärkt das Gefühl, nicht willkommen zu sein. Hinsichtlich einer eventuellen Binnenwanderung nach dem Studienabschluss wird, abgesehen von der Arbeitsmarktlage, der aktuelle Standort durch die bisherigen Akkulturationserfahrungen und die bereits entstandenen sozialen Netzwerke bevorzugt in Betracht gezogen.

\subsection{Verknüpfung von Migrationsentscheidungen mit biographischen Statuspassagen}

Die Kernhypothese dieses Beitrags lautet, dass bei der hier im Mittelpunkt stehenden Alterskohorte ein Verbleib bzw. eine weitere Migration nach dem Studienabschluss als integraler Bestandteil einer biographischen Statuspassage zu betrachten ist. Es ist anzunehmen, dass die Überlegungen hinsichtlich weiterer Mobilitätsschritte eng verknüpft sind mit Überlegungen zu den beruflichen Entwicklungsmöglichkeiten, Plänen zur Familiengründung oder familiären Verpflichtungen im Herkunftsland. Zudem sind die Überlegungen nicht auf Verbleib versus Rückkehr beschränkt, sondern überspannen verschiedene Staaten und jene Lebensbedingungen, die dort nach Einschätzung der Interviewpartner aktuell und in Zukunft vorzufinden sind.

Ein Beispiel für eine derart transnational angelegte Entscheidungsbasis ist die Art und Weise, wie GradM3, Pharmazie-Doktorand aus Äthiopien, zwischen wissenschaftlicher Karriere und ökonomischem Erfolg abwägt. Grundsätzlich würde er gerne in sein Heimatland zurück- 
kehren, um dort seine wissenschaftliche Karriere fortzusetzen. Angesichts der schwierigen Lebensbedingungen und eher schlechten Rahmenbedingungen für eine internationale wissenschaftliche Karriere antizipiert er aber auch eine Veränderung seiner Zukunftspläne und der damit verbundenen Standortentscheidungen: ,What I want is to stay in the academic area, and that's why I am actually doing my PhD. [...] For the time being, I am thinking to get back to Ethiopia and work, but [...] I've planned in previous days in different directions and I was forced to change many things. So things might change."

Auch bei StudW1, Bioinformatik-Studentin aus Rumänien, wird die transnationale Orientierung bei ihren Erörterungen von Karriereschritten und Berufserfahrungen deutlich: „Nach Rumänien möchte ich nicht unbedingt zurück gehen, weil ich mit Bioinformatik da nichts anfangen kann. [...] Ob ich jetzt in Deutschland bleibe, ich hab' das nicht unbedingt vor. Also ich schließe das nicht aus, aber ich mach' nicht draus mein Ziel. Was ich gerne machen würde, wäre mal, in die USA zu fahren und dort ein bisschen zu forschen, oder so, damit man sieht [...]. Also ich glaube, sie haben einfach andere Mentalität. Auch was das Forschen betrifft, und so. Und es würde mich interessieren, wie das aussieht."

Neben den beruflichen und ökonomischen Entwicklungsmöglichkeiten spielt auch die Frage der Übertragbarkeit des in Deutschland erworbenen Wissens eine Rolle. Während einige Studierende befürchten, ihr in Deutschland erworbenes Wissen nur schwer in den Herkunftskontext übertragen zu können, erwarten andere Vorteile durch ihre interkulturelle Orientierung, die sich besonders lukrativ in einem internationalen Unternehmen auszahlen könnten. So wünschen sich etliche Interviewpartner eine Beschäftigung bei einer deutschen Firma, die sie dann als Expatriate zu deutschen Konditionen in das jeweilige Heimatland entsenden könnte. Inwiefern sich diese Vorstellungen auch in die Tat umsetzen lassen, bleibt jedoch ungewiss, da keiner der Interviewpartner bislang Berufserfahrungen in Form von Praktika gesammelt hat.

Die eigenen Einschätzungen werden vielfach an den Verhaltensweisen der Gleichaltrigen im Herkunftsland gespiegelt. So dient der Hinweis auf die starken Auswanderungstendenzen der jungen Erwachsenen beispielsweise aus den postsozialistischen Transformationsstaaten Europas als Validierung der eigenen Haltung, nicht ins Herkunftsland zurückkehren zu wollen. Für manche stellt diese Auswanderungskultur den konkreten Verlust sozialer Netzwerke im Heimatland dar, sodass auch vor diesem Hintergrund eine Rückkehr nicht attraktiv erscheint und die neu gewonnenen sozialen Netzwerke am aktuellen Standort an Bedeutung gewinnen, so wie es StudM1 aus Brasilien formuliert: „Ich hab' mir neulich überlegt, will ich jetzt später in Deutschland bleiben oder in Brasilien? In Brasilien, also alle Leute, die eigentlich mein Leben bewegen, sind gerade hier, außer meiner Familie und ein paar Freunden, die ich in Brasilien habe, aber mit denen hab' ich auch nicht so viel Kontakt.“

Ein letzter Befund hinsichtlich der Schnittstelle von Migrationsentscheidung und biographischer Statuspassage ist der Wandel von Lebenszielen oder Gewichtungen der WorkLife-Balance und die Folgen für weitere Migrationsentscheidungen. Der interessanteste Einflussfaktor dürfte hierbei das Bedenken einer künftigen Familiengründung sein, wodurch Fragen der Lebensqualität und der Möglichkeiten, Beruf und Familie zu vereinbaren, an Tragweite zunehmen. Die Überlegungen werden auf den (potenziellen) Lebenspartner ausgedehnt, wobei im Falle von Partnern unterschiedlicher Herkunft die transnationale Komponente im Aushandlungsprozess noch verstärkt wird. Dies zeigt sich bei StudM6 aus den USA, der mit einer Deutschen verheiratet ist: „Possible that we stay here. But actually we want to move back to the U. S. in four to five years. [...] The health system in Germany is better, and also the education. But college seems to be better in the U. S. But you pay a lot of money for it. [...] We don't know where we'll actually end up. If we return to the U. S., it is not out of question that we re-emigrate to Germany one day."

Andere Interviewpartner antizipieren nicht nur die eigene Familiengründung, sondern auch Fürsorgeverpflichtungen gegenüber Familienangehörigen. Dies wurde besonders bei den asiatischen Interviewpartnern spürbar, die die $\mathrm{Be}$ deutung der familiären Bindung und Fürsorge für die eigenen Eltern als Grund für eine langfristige Rückkehr in das Heimatland heranzogen: „Später möchte ich mich um meine Eltern kümmern. Ja aber, ich bin momentan ledig, nicht, und bin so [...] das kommt darauf an, auf meinen zukünftigen Partner oder Situation meiner Eltern" (GradW4, Soziologin, Doktorandin, Japan).

Zusammenfassend lässt sich konstatieren, dass Erwägungen zu Lebenszyklus-Etappen und Standortentscheidungen stark miteinander verknüpft sind und auf der Basis eines transnationalen Aktionsraums angestellt werden. Die unterschiedlichen und manchmal widersprüchlichen Pläne und Wünsche hinsichtlich der Karriereentwicklung, des ökonomischen Wohlergehens, der Fürsorge für Familienangehörige und den Erfordernissen einer zukünftigen Familiengründung werden sorgfältig abgewogen und diskutiert. In den Argumentationsmustern finden sich sowohl ökonomische Rationalität, subjektive Wahrnehmungen, die Antizipation von herkunftsgesellschaftlichen Erwartungen sowie die Spiegelung der eigenen Vorstellungen am Peer-groupVerhalten. Über allem schwebt jedoch die Vorläufigkeit der Entscheidung, die sowohl in der fehlenden Abgeschlossenheit der aktuellen Lebenslauf-Etappe begründet ist wie auch in der Erfahrung, dass sich standortbezogene Einstellungen über die Zeit (z. B. im Rahmen eines Akkulturationsprozesses) verändern können. 


\section{Schlussbetrachtung}

Dieser Beitrag konzentrierte sich auf die Frage der Bleibeintentionen internationaler Studierender in Deutschland, die im Rahmen der deutschen Arbeitsmarkt- und Zuwanderungspolitik zunehmend als Potenzial zur Deckung des Fachkräftebedarfs betrachtet werden. Deutschland ist ein gefragtes Zielland für internationale Studierende und die formalen Rahmenbedingungen für einen Verbleib und Arbeitsmarktzugang nach Studienabschluss in den vergangenen Jahren wurden erheblich liberalisiert. Doch das allein ist kein Garant für hohe Verbleibszahlen. Umfrageergebnisse und Sekundärdaten zeichnen ein widersprüchliches Bild hinsichtlich des Verbleibs nach dem Studienabschluss: Einerseits wird ein großes Interesse am Verbleib geäußert, andererseits wird diese Intention in geringem Umfang in die Tat umgesetzt. Auf der Basis des Forschungsstandes zu Bleibeintentionen und deren wichtigsten Einflussfaktoren konzentrierte sich der Beitrag auf die Frage, wie sich die Verbleibs- oder Wanderungsentscheidung als Teil einer biographischen Statuspassage konstituiert, die am Übergang zwischen Studium und Beruf auftritt.

Ein biographisch orientierter methodischer Zugang ermöglichte es, die Aushandlungsprozesse der Studierenden in ihrer Komplexität zu untersuchen. Anhand der Reflexion der zurückliegenden Migrationsetappe konnte nachvollzogen werden, wie sich die Entscheidung in einer Mischung aus rationalen Erwägungen und Einflüssen aus sozialen Netzwerken konstituiert. Vorhergehende Auslandsaufenthalte erleichterten die Entscheidung in den meisten Fällen, häufig gab es dabei einen direkten Bezug zu Deutschland oder sogar zum aktuellen Standort. Es ist anzunehmen, dass auch für zukünftige Migrationsentscheidungen diese Komponenten zum Tragen kommen. Allerdings bedeutet die positive Reflexion des aktuellen Aufenthalts nicht automatisch, dass die Befragten die Standortvoraussetzungen auch für einen längerfristigen Aufenthalt als geeignet erachten. Denn hinsichtlich längerfristiger Bleibeentscheidungen spielen nicht nur die beruflichen Entwicklungsmöglichkeiten eine Rolle, sondern die Frage der sozialen Integration im Alltag tritt in den Vordergrund.

Dabei sind viele Faktoren, die die Entscheidung strukturieren, noch im Fluss, sodass der Argumentationsverlauf durchweg von Vorläufigkeit geprägt ist. Dieses Verhalten „bis auf Weiteres“ wird auch im Kontext der „zweiten Moderne" als markantes Merkmal der adoleszenten Generation konstatiert, die sich angesichts der sich ständig verändernden Rahmenbedingungen und einer Vielfalt von Optionen nicht mehr an den „Normalbiographien“ vorheriger Generationen orientieren können (vgl. Bauman 2000; Beck/BeckGernsheim 2002). Die hier untersuchte Gruppe zeigt Strategien, die biographischen Unsicherheiten, die in dieser Situation und an der vor ihnen liegenden Statuspassage auf- treten, zu neutralisieren. Ihr gelingt dies durch den Rückbezug auf eigene und familiäre Migrationserfahrungen und das Verhalten der peer group, sodass ein möglicherweise dauerhaftes transnationales Lebenskonzept als alternative Form einer „Normalbiographie“ entworfen wird.

Bei aller Vorläufigkeit der Selbstaussagen ist ein längerfristiger Verbleib in Deutschland für einen Teil der internationalen Studierenden wahrscheinlich, insbesondere wenn durch einen längeren Studienaufenthalt ein Akkulturationsprozess eingesetzt hat. Die Fallstudie zeigt jedoch, dass die Akkulturation nicht selbstverständlich im Rahmen des Aufenthaltes voranschreitet. Teilnehmer englischsprachiger Studienprogramme finden nicht zwangsläufig in den deutschen Alltag hinein, dieser wird jedoch im Falle eines längerfristigen Verbleibs von größerer Bedeutung sein als bei einem befristeten Aufenthalt. Ähnliche Konsequenzen sind von der lokalen Akkulturation zu erwarten, denn je stärker diese ausgeprägt ist, desto wahrscheinlicher würde ein Verbleib am Standort nach dem Studienabschluss angestrebt werden - unter der Voraussetzung adäquater Beschäftigungsmöglichkeiten.

Um die Bleibequote dieser hochqualifizierten internationalen Studierenden nach dem Studienabschluss zu erhöhen, bedarf es eines abgestimmten Vorgehens der Hochschulen, der kommunalen Verwaltung sowie der potenziellen Arbeitgeber. Eine breite Angebotspalette von Deutsch- und Integrationskursen, Transparenz hinsichtlich der Bleibebedingungen sowie die frühzeitige Heranführung an den Arbeitsmarkt und die Unterstützung des Übergangs vom Studium in den Beruf sind geeignete Möglichkeiten, um Unsicherheiten hinsichtlich der Zukunft in Deutschland abzubauen und die Bleibeentscheidung zu fördern. Was sicherlich nicht politisch verordnet werden kann, ist die gelebte Willkommenskultur im Alltag und die Akzeptanz von Diversität, was insbesondere in den bisher kaum von Zuwanderung erfassten Regionen im Osten Deutschlands noch entwicklungsbedürftig ist.

Es zeigt sich eindeutig weiterer Forschungsbedarf hinsichtlich der späteren Realisierung von ursprünglichen Migrations- oder Bleibeintentionen, was idealerweise in Form von Panel- oder Längsschnittstudien abzubilden wäre. Dabei wäre es wichtig, einen transnationalen Untersuchungsansatz zu wählen, um die Entscheidungs- und Handlungsmuster dieser hochmobilen und transnational orientierten Gruppe über die Zeit abzubilden. Von Interesse sind dabei nicht nur die Bleibe- oder Weiterwanderungsentscheidungen auf internationaler Ebene, sondern auch Entscheidungen hinsichtlich der Binnenmigration im Kontext regionaler Entwicklungsunterschiede und deren Perzeption. 


\section{Literatur}

Alberts, H. C.; Hazen, H. D. (2005): „There are always two voices“: International students' intentions to stay in the United States or return to their home countries. In: International Migration 43, 3, $131-154$.

Baláž, V.; Williams, A. M. (2004): 'Been there, done that': International Student Migration and Human Capital Transfers from the UK to Slovakia. In: Population, Space and Place 10, 3, 217-237.

Baldwin, G. B. (1970): Brain drain or overflow? In: Foreign Affairs 48, $2,358-372$

Bauman, Z. (2000): Liquid Modernity. Cambridge, Malden (MA).

Beck, U.; Beck-Gernsheim, E. (2002): Individualization: Institutionalized Individualism and its Social and Political Consequences. London.

BMI - Bundesministerium des Innern (2007): Migrationsbericht des Bundesamtes für Migration und Flüchtlinge im Auftrag der Bundesregierung. Migrationsbericht 2006. Berlin.

BMI - Bundesministerium des Innern (2008): Migrationsbericht des Bundesamtes für Migration und Flüchtlinge im Auftrag der Bundesregierung. Migrationsbericht 2007. Berlin.

BMI - Bundesministerium des Innern (2014): Migrationsbericht des Bundesamtes für Migration und Flüchtlinge im Auftrag der Bundesregierung. Migrationsbericht 2012. Berlin.

BMI - Bundesministerium des Innern (2016): Migrationsbericht des Bundesamtes für Migration und Flüchtlinge im Auftrag der Bundesregierung. Migrationsbericht 2014. Berlin.

Bourdieu, P. (1986): The Forms of Capital. In: Richardson, J. G. (Hrsg.): Handbook of Theory and Research for the Sociology of Education. New York, 241-260.

Boyd, M. (1989): Family and Personal Networks in International Migration: Recent Developments and New Agendas. In: International Migration Review 23, 3, 638-670.

Chiu, L.-I.C.; Hou, J.-Y. (2007): Determinants of Highly-Skilled Migration - Taiwan's Experiences. Taipei. = Chung-Hua Institution for Economic Research, Working Paper Series No. 2007-1. http://www.cier.edu.tw/public/Data/710161741071.pdf (21.08.2015)

DAAD - Deutscher Akademischer Austauschdienst; DZHW - Deutsches Zentrum für Hochschul- und Wissenschaftsforschung (2014): Wissenschaft Weltoffen 2014. Daten und Fakten zur Internationalität von Studium und Forschung in Deutschland. Bielefeld.

DAAD - Deutscher Akademischer Austauschdienst; DZHW - Deutsches Zentrum für Hochschul- und Wissenschaftsforschung (2015): Wissenschaft Weltoffen kompakt 2015: Daten und Fakten zur Internationalität von Studium und Forschung in Deutschland. http://www.wissenschaftweltoffen.de/kompakt/wwo2015 kompakt_de.pdf (21.08.2015).

Flick, U. (2007): Qualitative Sozialforschung - Eine Einführung. Reinbek bei Hamburg.

Galinski, D. (1986): Brain Drain aus Entwicklungsländern. Frankfurt am Main. = Europäische Hochschulschriften 5 .

Geddie, K. (2013): The Transnational Ties that Bind: Relationship Considerations for Graduating International Science and Engineering Research Students. In: Population, Space and Place 19, 2, 196-208.

Gibson, J.; McKenzie, D. (2009): The Microeconomic Determinants of Emigration and Return Migration of the Best and Brightest: Evidence from the Pacific. Bonn. = IZA Discussion Paper 3926. http://ftp.iza.org/dp3926.pdf (16.04.2016).

Hanganu, E.; Heß, B. (2014): Beschäftigung ausländischer Absolventen deutscher Hochschulen - Ergebnisse der BAMF-Absolventenstudie 2013. Nürnberg.

Heß, B. (2009): Bleiben hochqualifizierte Zuwanderer in Deutschland? Befragungsergebnisse des Bundesamtes für Migration und Flüchtlinge. In: Sozialwissenschaftlicher Fachinformationsdienst $2,11-30$.
Holzapfel, N. (2011): Gehälter für Absolventen. 70.000 Euro Einstiegsgehalt - kein Traum. In: Süddeutsche Zeitung online vom 9. November 2011. http://www.sueddeutsche.de/karriere/ gehaelter-fuer-absolventen-euro-einstiegsgehalt-kein-traum-1. 600035 (16.04.2016).

Le Bail, H.; Shen, W. (2008): The Return of the "Brains" to China: What are the Social, Economic and Political Impacts? Paris, Brüssel. = Asie Visions 11. https://www.ifri.org/sites/default/files/ atoms/files/AV11_ENG.pdf (Zugriff am 08.03.2016).

Martin-Luther-Universität (2008): Studierendenstatistiken. Unveröffentlicht. Halle an der Saale.

Mayer, M. M.; Yamamura, S.; Schneider, J.; Müller, A. (2012): Zuwanderung von internationalen Studierenden aus Drittstaaten. Nürnberg. $=$ Working Paper der Forschungsgruppe des Bundesamtes für Migration und Flüchtlinge 47.

Müller, C. M. (2007): Zur Bedeutung von Remigranten für Innovationsprozesse in China. Eine theoretische und empirische Analyse. Frankfurt am Main.

Neusel, A. (2012): Untersuchung der inter- und transnationalen Karrieren von WissenschaftlerInnen an deutschen Hochschulen. In: Die Hochschule 21, 1, 20-35.

Obermeier, T. (2014): Fachkräftemangel. Dossier „Arbeitsmarktpolitik“ der Bundeszentrale für politische Bildung. http:// www.bpb.de/politik/innenpolitik/arbeitsmarktpolitik/178757/ fachkraeftemangel? $\mathrm{p}=$ all (16.04.2016).

OECD - Organisation for Economic Co-operation and Development (2014): Education at a Glance 2014. Paris.

Pichler, E.; Prontera, G. (2012): Kulturelles Kapital auf dem Weg zur Professur. Unterschiedliche Ausgangslagen von Wissenschaftler/ innen mit Migrationshintergrund. In: Die Hochschule 1,91-101.

Pries, L. (2001): Internationale Migration. Bielefeld.

Saxenian, A. L. (2008): The international mobility of entrepreneurs and regional upgrading in India and China. In: Solimano, A. (Hrsg.): The international mobility of talent. Types, causes, and development impact. Oxford, 117-144.

Sezer, K.; Dağlar, N. (2009): Die Identifikation der TASD mit Deutschland - Abwanderungsphänomen der TASD beschreiben und verstehen. Krefeld.

Sheils, J. (Hrsg.) (2001): Gemeinsamer europäischer Referenzrahmen für Sprachen: lernen, lehren, beurteilen. München.

Soon, J.-J. (2008): The determinants of international students' return intention. University of Otago, Economics Discussion Papers DP_0806. Otago.

SVR - Sachverständigenrat deutscher Stiftungen für Integration und Migration (2011a): Migrationsland 2011. Jahresgutachten 2011 mit Migrationsbarometer. Berlin.

SVR - Sachverständigenrat deutscher Stiftungen für Integration und Migration (2011b): Vom internationalen Studierenden zum hoch qualifizierten Zuwanderer. Berlin.

SVR - Sachverständigenrat deutscher Stiftungen für Integration und Migration (2012): Mobile Talente? Ein Vergleich der Bleibeabsichten internationaler Studierender in fünf Staaten der Europäischen Union. Berlin.

SVR - Sachverständigenrat deutscher Stiftungen für Integration und Migration (2015): Zugangstor Hochschule. Internationale Studierende als Fachkräfte von morgen gewinnen. Berlin.

Szelényi, K. (2006): Students without borders? Migratory decisionmaking among international graduate students in the U.S. In: Knowledge, Technology \& Policy 19, 3, 64-86.

Teichler, U.; Janson, K. (2007): The Professional Value of Temporary Study abroad in Another European Country: Employment and Work of Former Erasmus Students. In: Journal of Studies in International Education 11, 3-4, 486-495.

Wolfeil, N. (2012): Auswirkungen des Auslandsstudiums auf spätere Mobilitäts- und Karrieremuster. Das Beispiel der polnischen Studierenden an deutschen Hochschulen. Wien. 